\title{
Design considerations for a background limited 350 micron pixel array using lumped element superconducting microresonators
}

\author{
Christopher M. McKenney ${ }^{a}$, Henry G. Leduc ${ }^{b}$, Loren J. Swenson ${ }^{a, b}$, Peter K. Day ${ }^{b}$, Byeong H. \\ $\operatorname{Eom}^{a}$, Jonas Zmuidzinas ${ }^{a}$ \\ ${ }^{a}$ California Institute of Technology,1200 East California Blvd,Pasadena,United States; \\ ${ }^{b}$ NASA Jet Propulsion Laboratory, 4800 Oak Grove Drive,Pasadena, United States
}

\begin{abstract}
Future submillimeter telescopes will demand arrays with $\sim 10^{6}$ pixels to fill the focal plane. MAKO is a $350 \mu \mathrm{m}$ camera being developed to demonstrate the use of superconducting microresonators to meet the high multiplexing factors required for scaling to large-format arrays while offering background-limited single-pixel sensitivity. Candidate pixel designs must simultaneously meet many requirements. To achieve the desired noise equivalent powers it must efficiently absorb radiation, feature a high responsivity, and exhibit low intrinsic device noise. Additionally, the use of high resonator quality factors of order $\sim 10^{5}$ and resonant frequencies of order $f_{\text {res }} \approx 100 \mathrm{MHz}$ are desirable in order to reduce the per-pixel bandwidth to a minimum set by telescope scan speeds. This allows a maximum number of pixels to be multiplexed in a fixed electronic bandwidth. Here we present measurement results of the first MAKO prototype array which meets these design requirements while demonstrating sufficient sensitivity for background-limited operation at ground-based, far-infrared telescopes.
\end{abstract}

Keywords: lumped-element, superconductivity, kinetic-inductance, far-infrared, titanium nitride

\section{INTRODUCTION}

MAKO is a $350 \mu \mathrm{m}$ camera being developed as a pathfinder instrument to demonstrate superconducting microresonator detectors which meet the requirements for large-scale arrays of background limited pixels. The proposed CCAT 25-meter submillimeter telescope ${ }^{1}$ would require $\sim 10^{6}$ pixels at $\lambda=350 \mu \mathrm{m}$ to completely fill the focal plane. MAKO will use lumped element kinetic inductance detectors ${ }^{2}$ (LEKID) fabricated using a TiN superconducting microresonator. An historical overview and detailed description of motivation for this are outlined in another article in these proceedings. ${ }^{3}$

The inductor of the LEKID acts as an absorber of far infrared radiation, breaking Cooper pairs and generating quasiparticles in the resonator which will affect the surface impedance of the inductor, $\sigma=\sigma_{1}-j \sigma_{2}$. The MattisBardeen equations describe the changes in surface impedance to quasiparticle density, ${ }^{4}$

$$
\begin{aligned}
\frac{\sigma_{1}}{\sigma_{N}} & =\frac{2 \Delta}{\hbar \omega} \frac{n_{q p}}{N_{0} \sqrt{2 \pi k_{B} T \Delta}} \sinh (\xi) \mathrm{K}_{0}(\xi) \\
\frac{\sigma_{2}}{\sigma_{N}} & =\alpha \gamma \frac{\pi \Delta}{\hbar \omega}\left(1-\frac{n_{q p}}{N_{0} \Delta}\left(1+\sqrt{\frac{2 \Delta}{\pi k_{B} T}} e^{-\xi} \mathrm{I}_{0}(\xi)\right)\right)
\end{aligned}
$$

where $2 \Delta$ is the Cooper pair energy, $N_{0} \sim 8.7 \times 10^{9} \mathrm{eV}^{-1} \mu \mathrm{m}^{-1}$ is the single spin electron density of states estimated for $\operatorname{TiN}^{5}, \xi=\hbar \omega / 2 k_{B} T$, and $\omega$ the readout frequency. Changes in the surface impedance will result in a change in both the resonator dissipation and frequency. The quasiparticle density can be estimated by considering both the generation and recombination rate. Quasiparticles will be generated from absorbed radiation at a rate $\Gamma_{o p t}=\eta_{0} P_{\text {opt }} / \Delta$, where $\eta_{0}$ is a conversion efficiency $\sim 0.7, P_{\text {opt }}$ is the absorbed optical power,

Further author information: (Send correspondence to C.M.M)

C.M.M.: E-mail: chrism@astro.caltech.edu

Millimeter, Submillimeter, and Far-Infrared Detectors and Instrumentation for Astronomy VI, edited by Wayne S. Holland, Jonas Zmuidzinas, Proc. of SPIE Vol. 8452

84520S @ 2012 SPIE - CCC code: 0277-786X/12/\$18 - doi: 10.1117/12.925759

Proc. of SPIE Vol. 845284520 S-1 
and $\Delta$ is the superconducting gap parameter. Quasiparticle lifetimes in many superconductors are observed to follow

$$
\tau_{q p}=\frac{\tau_{\max }}{1+\frac{n_{q p}}{n^{*}}}
$$

where $n^{*} \sim 100 \mu \mathrm{m}^{-3}$ is a typical value and $\tau_{\max } \propto T_{c}^{-2}$ is the maximum quasiparticle lifetime observed at low temperatures and with no optical load. Measurements of quasiparticle lifetimes in TiN have shown reasonable agreement with the $T_{c}^{-2}$ scaling. ${ }^{5}$ Balancing generation and recombination rates leads to a quasiparticle density under load,

$$
n_{q p}=\sqrt{\left(n_{q p, t h e r m}+n^{*}\right)^{2}+2 n^{*} \frac{\tau_{\max } P_{o p t} \eta_{0}}{\Delta V}}-n^{*}
$$

where $V$ is the volume of the device. Together with the Mattis-Bardeen equations, 1a and $1 \mathrm{~b}$, this equation allows us to calculate the detector responsivity as a function of the various parameters. The choice of superconductor will affect the material parameters; a simple BCS model predicts $2 \Delta=3.52 k_{B} T_{c}$. In the regime where the quasiparticle density is dominated by optically generated quasiparticles we find $n_{q p} \propto T_{c}^{-5 / 2}$. An advantage to TiN is that $T_{c}$ can be varied from $0.8 \mathrm{~K}$ to $4 \mathrm{~K}$ by adjusting the nitrogen content during deposition allowing the $T_{c}$ to be controlled to meet the NEP and readout requirements. ${ }^{5}$

The NEP when measuring perturbations in the frequency and dissipation of the resonator have been presented in a review paper, ${ }^{6}$

$$
\begin{aligned}
& \mathrm{NEP}_{\text {diss }}^{2}=2 P_{o p t} h \nu\left(1+n_{0}\right)+N E P_{g-r}^{2}+\frac{8 n_{q p}^{2} V^{2} \Delta_{0}^{2}}{\eta_{0}^{2} \chi_{c} \chi_{q p}^{2} \tau_{q p}^{2}} \frac{k_{B} T_{a m p}}{P_{a m p}} \\
& \mathrm{NEP}_{\text {freq }}^{2}=2 P_{o p t} h \nu\left(1+n_{0}\right)+N E P_{g-r}^{2}+\frac{8 n_{q p}^{2} V^{2} \Delta_{0}^{2}}{\beta^{2} \eta_{0}^{2} \chi_{c} \chi_{q p}^{2} \tau_{q p}^{2}} \frac{k_{B} T_{a m p}}{P_{a m p}}+\frac{8 n_{q p}^{2} V^{2} \Delta_{0}^{2} Q_{i}^{2}}{\beta^{2} \eta_{0}^{2} \chi_{q p}^{2} \tau_{q p}^{2}} S_{T L S}
\end{aligned}
$$

The first contribution to each term in Eq. 4a-4b is the photon shot noise. Operation at the CalTech Submillimeter Observatory (CSO) will likely be performed under single polarization optical powers ranging from $25-50 \mathrm{pW}$ at $\lambda=350 \mu \mathrm{m}$ yielding a background limited NEP $\sim 4-5 \times 10^{-16} \mathrm{~W} / \sqrt{\mathrm{Hz}}$. The second term arises from generation-recombination noise but will not be a significant source if $h \nu \gg \Delta$. The third term in both dissipation and frequency is related to the amplifier noise, however in the frequency direction the response is enhanced by a factor $\beta$ over the dissipation direction and $\beta$ increases as the readout frequency is reduced. ${ }^{6}$ The dominant noise in the detector results from capacitance fluctuations due to two level systems (TLS). This term also includes the factor of $\beta$, further motivating lower frequency operation.

\section{PIXEL DESIGN: CONSIDERATIONS AND CONSTRAINTS}

The basic layout of the LEKID design used for MAKO is shown in cartoon form in Fig. 1(a). The absorber area is set by the Nyquist sampling criterion and the optics for operation at the CSO, $A_{L}=(f \lambda / 2)^{2} \approx 0.8 \times 0.8 \mathrm{~mm}^{2}$. A resonance frequency near $100 \mathrm{MHz}$ is desirable for reducing readout electronics cost, and we also require resonator $Q$ 's around $10^{5}$ to achieve high multiplexing density. Finally the pixel must be scalable to a large array format and achieve background limited NEPs under single polarization optical loads of $25-50 \mathrm{pW}$.

\subsection{Radiation Absorption}

Obtaining a low NEP requires maximizing the response of the device to incident $350 \mu \mathrm{m}$ radiation. TiN has a high normal-state resistivity $\rho_{N} \approx 100 \mu \Omega \mathrm{cm}$ and has been very successfully used as an absorber for farinfrared bolometers. ${ }^{7}$ An absorber illuminated through the back of a dielectric slab can achieve near unity optical efficiency if an anti-reflecting coating and quarter wave backshort are used and if the sheet resistance is matched to

$$
R_{s, e f f}=\frac{\eta_{0}}{\sqrt{\epsilon_{r}}}
$$




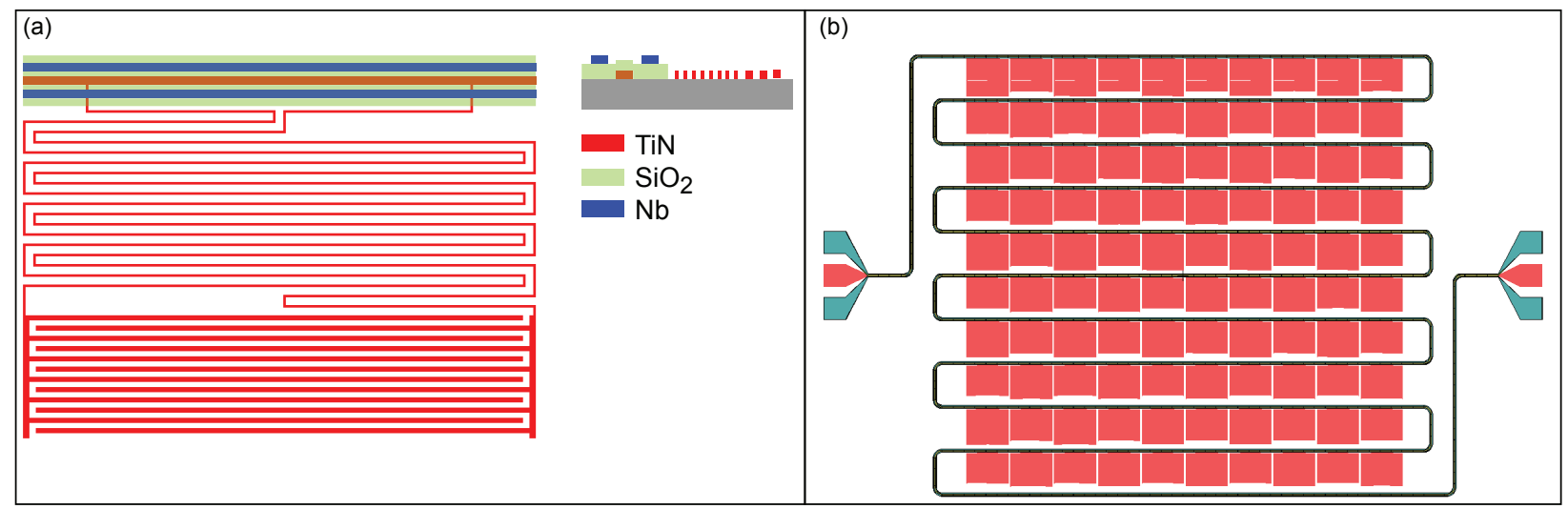

Figure 1. Cartoon layouts of the initial MAKO pixel tests. (a) Cartoon of a single pixel (not to scale). The pixel is fabricated in three lithography layers. TiN is deposited and etched away to define a center conductor for a co-planar wave-guide, a wrapped meander inductor and an interdigitated capacitor. $\mathrm{An} \mathrm{SiO}_{2}$ dielectric layer is deposited, then $\mathrm{Nb}$ ground lines for the CPW are defined on top of this. (b) Layout of the CPW and pixels in a 100 pixel array. All pixels have identical meander absorbers and the $L C$ readout frequency is varied by changing the capacitor geometry.

where $\eta_{0} \approx 377 \Omega$ is the impedance of free space and $\epsilon_{r}$ the relative permitivity of the dielectric. For a Si dielectric $\left(\epsilon_{r} \approx 11.8\right)$ the optimal surface impedance from Eq. 5 is $R_{s, \text { eff }} \approx 110 \Omega$. In the LEKID design adjusting the width and spacing of the meander inductors allow the effective sheet resistance to be increased. So long as the separation (s) between traces satisfies $s \ll \lambda$, the effective sheet resistance seen by the incident radiation will be

$$
R_{s, e f f}=\frac{\rho_{N}}{\phi t}
$$

where $\phi=w /(w+s)$ is the filling fraction and $\rho_{N}$ the normal resistivity of the superconductor. TiN is well suited to meet this requirement; for $w=1 \mu \mathrm{m}$ the filling fractions required to meet the impedance matching conditions are $\approx 0.1-0.5$ for film thicknesses of $20-100 \mathrm{~nm}$. In elemental superconductors such as Al the resistivity is typically two orders of magnitude lower, making it difficult to effectively impedance match the absorber at $\lambda=350 \mu \mathrm{m}$.

The volume is an important parameter since it controls the density of optically generated quasiparticles. Equating the resistances in Eq. 5 and Eq. 6 results in a fixed volume for a given absorbing area,

$$
V=A_{L} \phi t=\frac{A_{L} \rho_{N}}{\eta_{0}}\left(\sqrt{\epsilon_{r}}\right)
$$

For a Si dielectric and TiN absorber with the absorber area set by the CSO optics $\left(\approx 0.65 \mathrm{~mm}^{2}\right)$ this gives $V \approx 0.6 \times 10^{4} \mu \mathrm{m}^{3}$.

\subsection{Low Frequency Operation}

High multiplexing density requires resonators with low readout frequencies, so the goal we have set is $f_{\text {res }} \sim$ $100 \mathrm{MHz}$. An additional benefit of operation at lower readout frequencies is significant reduction in the per-pixel readout cost which will be beneficial in moving to high pixel arrays. ${ }^{3}$ To achieve $L C$ resonance frequencies at these values a large inductance is needed. Fortunately TiN has a high kinetic inductance: the Mattis-Bardeen theory gives

$$
L_{s}=\frac{\hbar}{\pi \Delta} R_{N}
$$

and for $\mathrm{TiN}$ with $T_{c} \approx 1 \mathrm{~K}$, trace widths $\sim 1 \mu \mathrm{m}$, and film thicknesses $\sim 50 \mathrm{~nm}$ the sheet inductance can easily exceed $20 \mathrm{pH} / \mathrm{sq}$. For a fixed absorber area $A_{L}$ the inductor has a total kinetic inductance $L_{k i n}=L_{s q} \times A_{L} \phi / w^{2}$ which gives a resonant frequency scaling as $f_{\text {res }} \propto 1 / w$. Our lithography limits line widths and spaces to $\approx 0.5 \mu \mathrm{m}$ and the thinnest reliable layers to $\sim 20 \mathrm{~nm}$. 


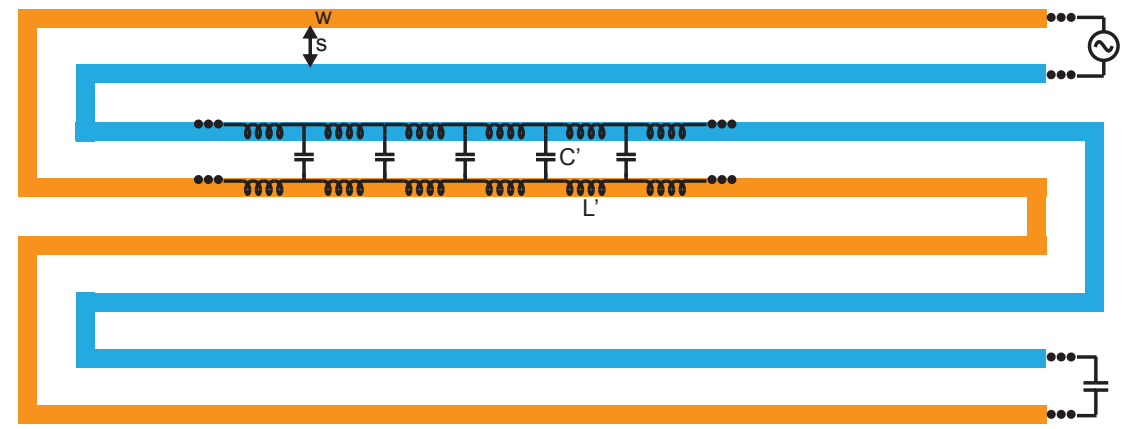

Figure 2. Transmission line model of the double wound meander inductor which behaves in a manner similar to a CPS at higher frequencies. The inductance per unit length is dominated by the kinetic inductance of the TiN, while the large capacitance comes from the double-wrapped meander shaped used to reduce inter-pixel crosstalk. The LEKID is terminated by an interdigitated capacitor which for the geometries considered here can be treated as a lumped element to frequencies $\sim 10 \mathrm{GHz}$.

\subsection{Pixel Crosstalk and Multiplexing Bandwidth}

Ultimately the MAKO architecture must scale to a large number of pixels while efficiently covering the focal plane. Efficient coverage requires pixels being geometrically tightly packed across the focal plane and operation of a large number of pixels within limited multiplexing bandwidth requires the resonators to be closely packed in frequency space as well. This high pixel packing density can result in electronic crosstalk between pixels arising from the electromagnetic coupling between resonators. ${ }^{8}$ To reduce the crosstalk, the meander inductor uses the double wound geometry shown in Fig. 1 where conductors of opposite polarity are in close proximity. To further reduce crosstalk the MAKO array uses a similar scheme to that used by Noroozian et. al ${ }^{8}$ whereby the resonators are split into different frequency bands and distributed physically in a pattern ensuring that no nearest neighbors are within the same frequency readout band.

While this design reduces cross talk the double wound geometry comes at a price of increasing the capacitance within the absorber meander. As the size of the resonator capacitor is reduced, the parasitic capacitance of the meander inductor will become important, making it necessary to model the lumped inductor as a transmission line with a geometry very similar to that of a co-planar stripline (CPS). This will have two consequences which degrade device performance. The first is that the current distribution within the inductor will become nonuniform in a manner which depends on the terminating capacitor for each resonator, this will result both in lower responsivity and non-uniform responsivity across the array.

The second consequence will be an unwanted resonance at a frequency corresponding to a half an effective wavelength in the resonator. For the CPS model the inductance per unit length can be approximated from the kinetic inductance $L^{\prime} \approx 2 \times L_{s q} / w$. The capacitance per unit length is roughly half of that of an equivalent interdigitated capacitor, thus $C^{\prime} \approx \frac{\epsilon_{e f f} \epsilon_{0}}{2} \frac{K(k)}{K^{\prime}(k)}$ where $K$ and $K^{\prime}$ are elliptic integrals depending on the fill factor of the inductor, $k=(\tan (\phi \pi / 4))^{2}$, and the ratio $K / K^{\prime}$ is typically of order unity. This model is shown in Fig. 2. The total length of the CPS will depend on the selected width of the trace lines as well as the filling fraction, $l_{L}=A_{L} \phi / 2 w$ and therefore a half-wave resonance will occur at $f_{\lambda / 2}=1 /\left(2 \pi \sqrt{L^{\prime} C^{\prime}}\right)$. The total available multiplexing bandwidth will be given by the ratio between this resonance and the $L C$ resonant frequency,

$$
\frac{f_{\lambda / 2}}{f_{L C}}=\pi \sqrt{\frac{C_{I D C}}{l C^{\prime}}}
$$

Several devices were modeled using various trace widths and filling fractions. For trace widths $\sim 1 \mu \mathrm{m}$ the typical ratio is $\sim 6-7$, this meets the MAKO requirement of $1-2$ octaves of multiplexing bandwidth.

\subsection{Capacitor Requirements and TLS Noise}

In the LEKID design the capacitor absorbs photons but does not produce any significant response, motivating a design with the smallest geometrical capacitor footprint which still meets the NEP and multiplexing requirements. 


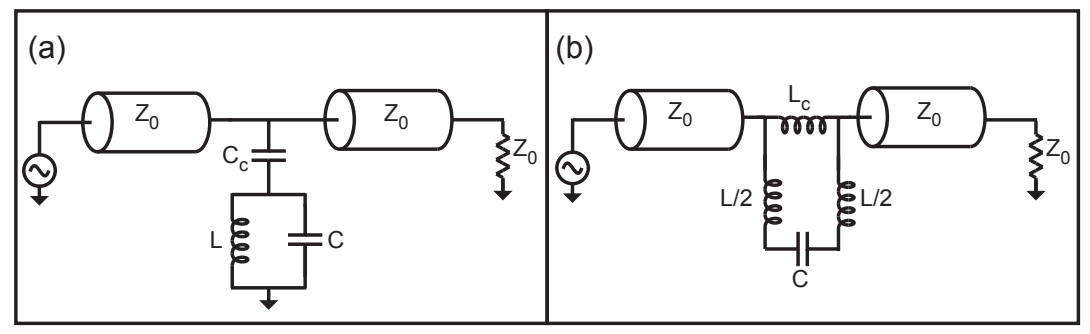

Figure 3. $L C$ circuit configurations and their respective coupling schemes to the RF transmission line. (a) A capacitive coupling scheme is shown with parallel $L C$ circuit. (b) The inductive coupling scheme with series $L C$ circuit, this is the the equivalent circuit used in the MAKO devices.

Future devices may use microlens arrays which will focus the radiation only on the sensitive portions of the resonators; the application to these lens arrays is discussed later. For the MAKO camera we are initially planning to demonstrate bare pixels, without lenses.

The capacitor must be designed to provide an $L C$ frequency $\sim 100 \mathrm{MHz}$. For the typical geometries described here the kinetic inductance of TiN allows $L_{k i n}>10^{3} \mathrm{nH}$, so for an $L C$ frequency of $100 \mathrm{MHz}$ this requires $C \sim 5 \mathrm{pF}$. For a fixed capacitor area the total $C$ will increase approximately as the total number of fingers which pushes the design toward narrower traces. To achieve the capacitances needed while not exceeding half of the available area, trace widths and spacings of $\sim 1-2 \mu \mathrm{m}$ are required.

In Eq. 4b the NEP has a term in the frequency direction arising from two level systems (TLS) noise in the capacitor. This noise arises from an amorphous dielectric surface layer near the electrodes of the capacitor such as a surface oxide or adsorbed layer, and can be reduced by separating the capacitor fingers or increasing the total area of the capacitor ${ }^{4}$.

\subsection{Resonator $Q$ and RF Coupling}

The $L C$ resonator circuit is coupled to the transmission line with some coupling strength $Q_{c}$ set by the geometry of the feed line and coupling structure. In the MAKO device an inductive scheme is used, where the resonator is driven directly by galvanically coupling the inductor to the center conductor of the CPW feedline. The coupling $Q_{c}$ in this case is

$$
Q_{c, i n d}=2 Z_{0}\left(\frac{1}{\omega L_{c}}\right)\left(\frac{L}{L_{c}}\right)=\frac{2 Z_{0}}{Z_{\text {res }}}\left(\frac{L}{L_{c}}\right)^{2}
$$

where $Z_{\text {res }}=\sqrt{L / C}$ is the characteristic impedance of the resonator and for our devices is $\approx 200 \Omega$. In the case of inductive coupling the coupling inductor $L_{c}$ is determined by the both the inductance per unit length of the transmission line and the length between the coupling fingers.

For the MAKO design our goal is to design a coupling $Q_{c} \approx 250 \times 10^{3}$ for devices with optically loaded $Q_{i}$ 's $\approx 200 \times 10^{3}$. Sonnet simulation software was used to estimate the inductance per unit length $\left(L^{\prime}\right)$ of the transmission line. The spacing between the coupling legs is calculated using Eq. 10,

$$
l=\frac{L}{L^{\prime}} \sqrt{\frac{Q_{c}}{2} \frac{Z_{r e s}}{Z_{0}}}
$$

Each pixel uses the same meander inductor and transmission line so variation in the required spacing depends only on the interdigitated capacitor, $l \propto \sqrt{Z_{\text {res }}} \propto C^{-1 / 4}$. Large changes in capacitance result in only small required changes in the leg spacing to achieve the same $Q_{c}$ which allows uniform coupling to be easily designed using this geometry. The capacitance is varied to set the resonant frequency of each resonator, for two octaves this will require changing the leg spacing by only $\approx 40 \%$. 


\subsection{Device responsivity and selection of $T_{c}$}

The expected quasiparticle density as a function of absorbed optical power is given by Eq. 3 . While the volume is constrained (Eq. 7) the $T_{c}$ of the device can be tuned to achieve the required resonator $Q$ and responsivity. As discussed earlier in the regime where optically generated quasiparticles dominate over thermally generated quasiparticles the quasiparticle density scales as $n_{q p} \propto T_{c}^{-5 / 2}$, so the responsivity increases rapidly as $T_{c}$ is reduced. However, as $n_{q p}$ increases the resonator $Q$ decreases which reduces the multiplexing density that is achievable.

The optical responsivity will be significantly degraded when the density of thermally generated quasiparticles becomes comprable to the density of optically generated quasiparticles. The MAKO cryostat has a base temperature around $220 \mathrm{mK}$. The thermal quasiparticle density from BCS theory $\left(n_{q p, t h}=2 N_{0} \sqrt{2 \pi k_{B} T \Delta e^{-\Delta / k_{B} T}}\right)$ predicts that for a typical operating temperature of $250 \mathrm{mK}$ the density of thermally generated quasiparticles will approach the density of optically generated quasiparticles in Eq. 3 for $T_{c} \approx 1 \mathrm{~K}$, which sets an approximate lower limit to the $T_{c}$ and an upper limit on the optical responsivity.

\subsection{Amplifier Noise}

The NEP contribution from the amplifier in Eq. 4a-b can be most easily reduced by increasing the readout power. Unfortunately there exists some resonator energy at which point the nonlinear kinetic inductance effect causes the resonator to have a bifurcated, hysteretic response. While we have experimentally found that it is possible to obtain excellent NEP even when operating in the bifurcation regime, for MAKO we wish to avoid the complications in readout protocols that this entails. The relevant scale $E_{*}$ for the stored energy in the resonator at which bifurcation occurs is found to be related to the condensation energy of the superconductor $E_{c}=2 N_{0} \Delta^{2} V ;$ the corresponding readout power is ${ }^{3}$

$$
P_{g, b i f} \approx 0.8 \frac{Q_{c} \omega_{r} E_{*}}{2 Q_{r}^{3}}
$$

In practice we seem to measure bifurcation at a few $\mathrm{dB}$ above the value predicted using $E_{*}=E_{c}$ across a wide range of devices, ${ }^{3}$ but due to difficulties in measuring the attenuation and cryogenic losses it is difficult to measure exactly the feedline power to within more than a few $\mathrm{dB}$. Operating at a higher $T_{c}$ and larger volume increases the allowable readout power, but the device volume is fixed by the radiation matching requirements and $T_{c}$ is set by both the desired responsivity and internal $Q$ needed for mulitplexing. Ultimately these other constraints will define the geometry and $T_{c}$ which fixes the maximum readout power.

\section{INITIAL DEVICE TESTING}

The initial device design is shown in Fig. 4. For the first generation of MAKO pixels the absorber area is $A_{L}=1 \times 0.6 \mathrm{~mm}^{2}$ and the capacitor area $A_{C}=1 \times 0.4 \mathrm{~mm}^{2}$, giving a $60 \%$ fill fraction for the focal plane. The arrays were patterned in a $10 \times 10$ grid with the number of capacitor fingers varied to adjust the resonant frequency of each resonator. For ease of fabrication the overall pixel was kept square, leading to a rectangular inductor/absorber section, which is acceptable since this first-generation device is intended to test feasibility only and will not be used for imaging. Two sets of capacitor geometries were designed with finger widths and spacings of 1 and $2 \mu \mathrm{m}$ respectively. The absorbing inductor has a trace width $w=2 \mu \mathrm{m}$ and spacing $s=4 \mu \mathrm{m}$; for $\rho_{N}=100 \mu \Omega \mathrm{cm}$ this yields an effective sheet resistance $R_{s} \approx 70 \Omega$. The device is mounted without an anti-reflective (AR) coating or quarter wave backshort. With an AR coating and quarter wave backshort a well matched device would achieve near unity absorption; without either of these the optical efficiency of the present device should be $\sim 50 \%$.

The devices were tested in two available dilution refrigerators. A cryogen free dilution refrigerator allowed greater throughput and was used extensively for yield and readout measurements, while a liquid helium system with a blackbody and $\lambda=215 \mu \mathrm{m}$ filter was used for measuring the optical response, internal $Q$, and noise. The

blackbody was kept at temperatures below $35 \mathrm{~K}$, therefore the photon occupation number contributing to the photon shot noise NEP in Eqs. 4a and 4b will be small compared to on sky operation at the CSO. 

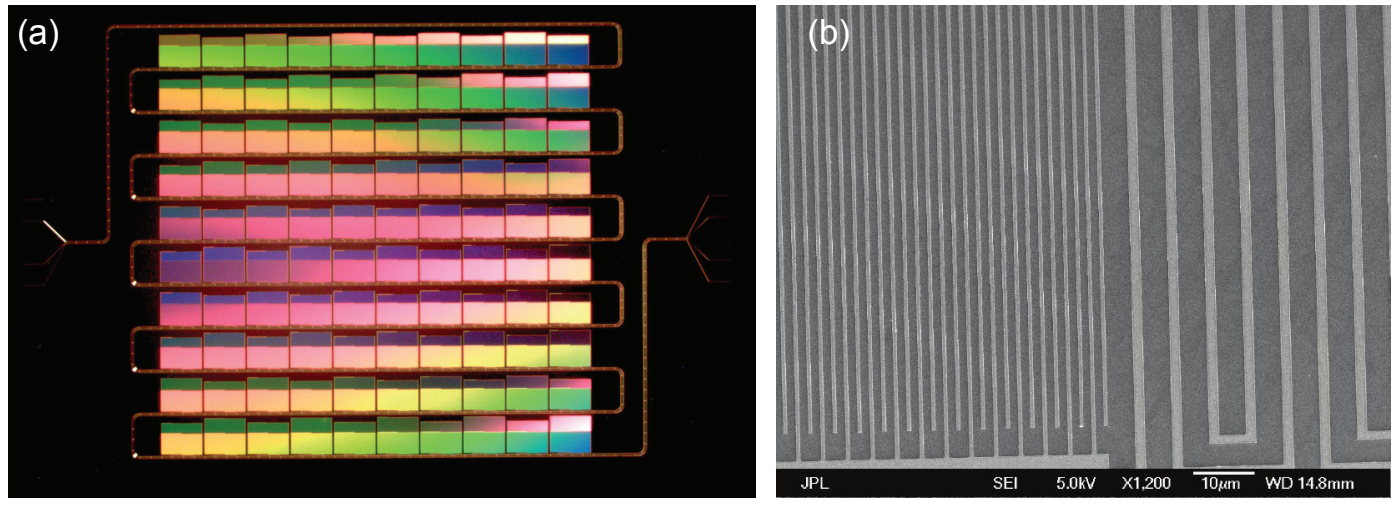

Figure 4. (a) Optical microscope photo of 100 pixel array device. The readout frequency of the detectors is determined by the length of the capacitor. The CPW couples through all devices as it meanders between the two bondpads. Ground straps are visible across the CPW center conductor. (b) SEM image of a device showing the meander inductor and capacitor. For this device the meander was designed to have $2 \mu \mathrm{m}$ traces and $4 \mu \mathrm{m}$ separation and the capacitor designed for $1 \mu \mathrm{m}$ widths and $1 \mu \mathrm{m}$ separation.

\subsection{Quality Factor}

To test the inductive coupling scheme, a 100 pixel array was fabricated using a $T_{c} \approx 4 \mathrm{~K}$ TiN film. The inductive coupling was designed using Eq. 11 with a goal of achieving $Q_{c}=2.5 \times 10^{5}$. Of the 100 designed resonators we had a yield of 75 resonators, and yields of $\approx 80 \%$ have been typical for test devices so far. The RF transmission (S21) was measured at powers well below bifurcation to fit $Q_{c}$ and the results are shown in Fig. 5(a). The mean $Q_{c} \approx 3 \times 10^{5}$ was somewhat higher than the designed value. The real benefit of this design is the uniformity of $Q_{c}$ as shown in Fig. 5 , this level of nonuniformity would result in negligibly small variations in NEP across the array. Changing the $T_{c}$ of the device caused $Q_{c}$ to vary as expected, for devices with $T_{c}=1.2 \mathrm{~K}$ we measure $\left\langle Q_{c}\right\rangle \approx 5.6 \times 10^{5}$.
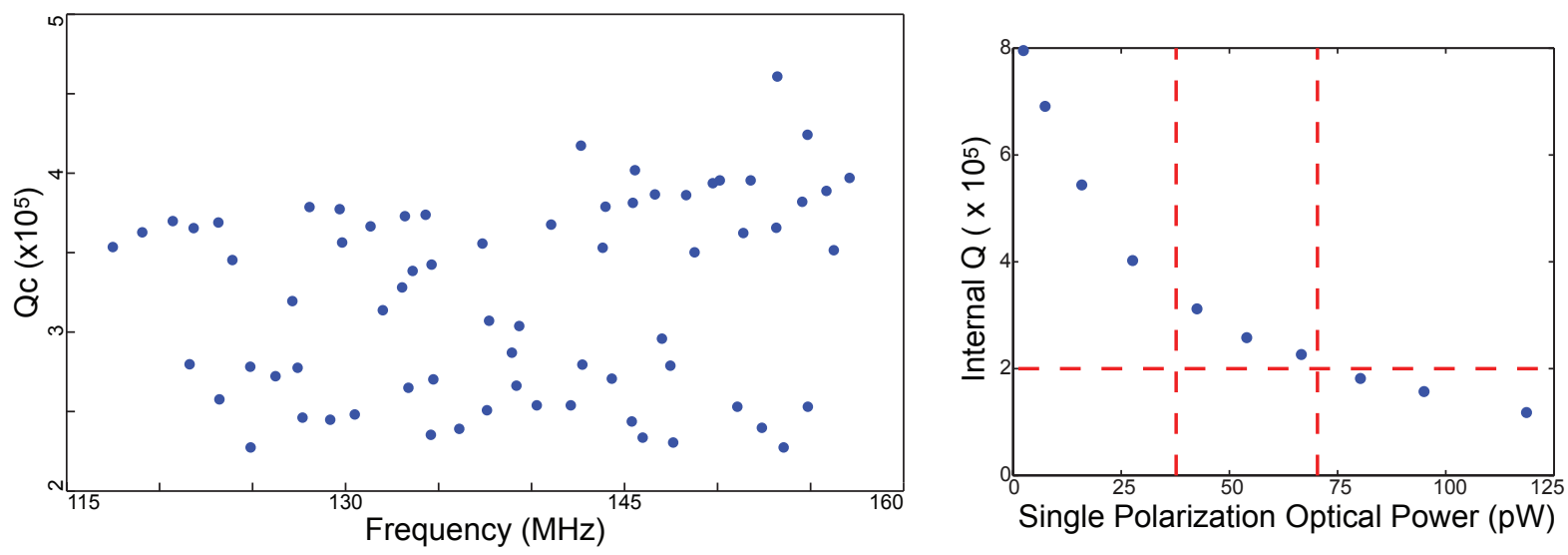

Figure 5. (a) Fit $Q_{c}$ from the measured S21 transmission on a 100 pixel test array. The test array had $T_{c} \approx 4 K$. The design goal was $Q_{c}=2.5 \times 10^{5}$, here we note a slight offset from the mean and a small scatter $\left(\sigma_{Q_{c}} \approx 6.8 \times 10^{4}\right)$ in the coupling value. (b) Fit of internal $Q$ on a test pixel with $T_{c}=1.2 \mathrm{~K}$ under blackbody load. The horizontal dashed line is the minimum $Q_{i}$ desired to meet multiplexing requirements. The vertical dashed lines correspond to the low and high range of loading respectively expected for operation at the CSO.

To estimate the internal $Q$ under loading conditions a $T_{c}=1.2 \mathrm{~K}$ pixel array was fabricated and measured in a dilution refrigerator with a blackbody load. The measured internal quality factor $Q_{i}$ of a single resonator is shown in Fig. 5(b), in the expected range of singe polarization optical powers $(25-50 \mathrm{pW})$ it is $\sim 2-3 \times 10^{5}$. 
These devices meet the required resonator $Q$ s for multiplexing needs.

\subsection{Frequency Responsivity}

The fractional frequency responsivity $\left(R=d x / d P ; d x=\delta f / f_{0}\right)$ of the devices with $T_{c} \approx 1.2 \mathrm{~K}$ was measured under blackbody load. Initially the measured responsivity increased significantly at high optical load, whereas Eq. 3 predicts the responsivity should decrease with the square root of the optical power. One obvious possibility was that the optical power was heating the chip and generating thermal quasiparticles. To test this a second device was made with a $\mathrm{Au}$ border film around the perimeter with a width of $500 \mu \mathrm{m}$ and thickness of $350 \mathrm{~nm}$ that could be wire bonded to the sample box. We first measured the response to blackbody radiation with $\sim 20$ wire bonds and noticed a significant change in the shape of the response curve as well as a significant decrease in the responsivity at high powers. However the response still flattened out and had a minor upturn at the highest optical powers. This is not fully understood and additional tests including larger thermalization areas are under way to try to understand this behavior.

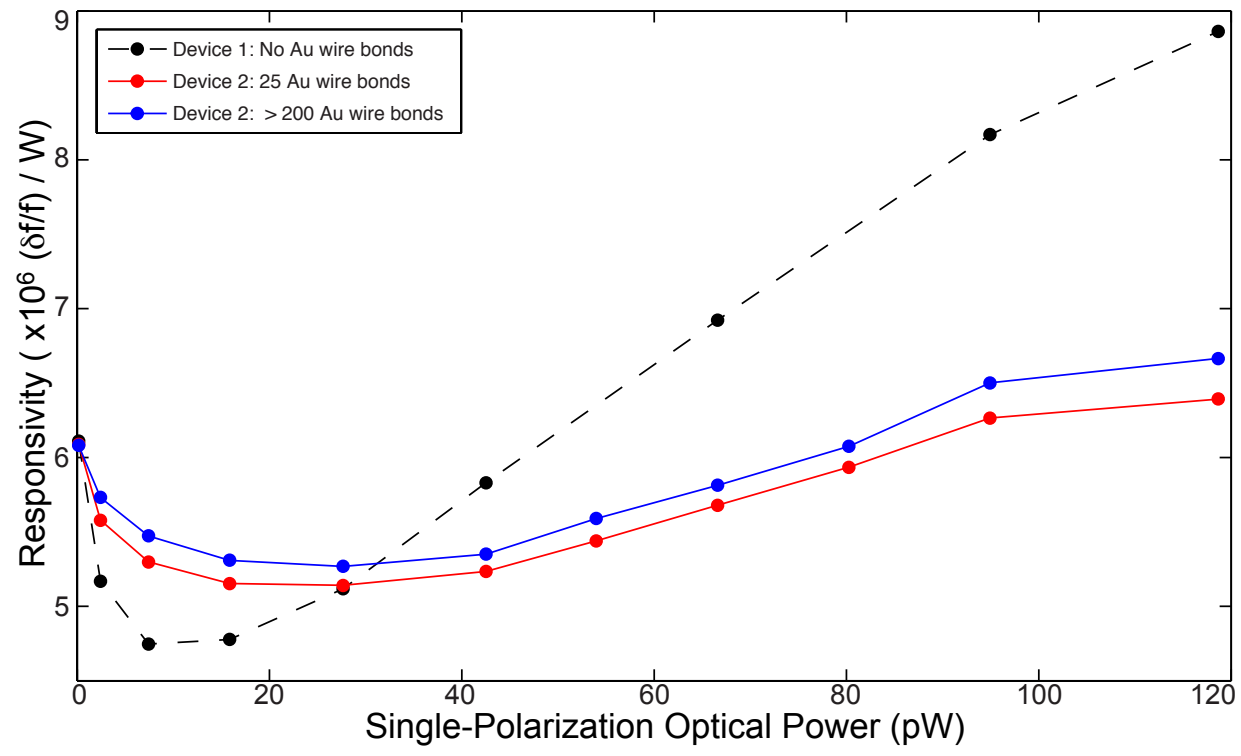

Figure 6. Responsivity of a $T_{c}=1.2 \mathrm{~K}$ MAKO pixel to blackbody load. The single polarization optical power is the calculated power falling on the inductor meander based on the geometry of the blackbody and filter used and ignores optical efficiency of the device. There is a significant change in the shape when an Au pad and wire bonds are added for thermal heat sinking. The shape of the curve does not change very much when the wire bond density is increased by an order of magnitude, the small offset between these two curves is well within the uncertainty in optical power introduced by uncertainty in the geometrical placement of the blackbody between measurements.

\subsection{Noise}

The noise for the $T_{c}=1.2 \mathrm{~K}$ device was measured at various mixing chamber temperatures and under various blackbody loads. Under no optical loading the dominant noise appears to be from TLS. At the lowest noise frequencies $(\nu<1 \mathrm{~Hz})$ temperature fluctuations in the dilution refrigerator introduce noise from thermal quasiparticles, and we were able to operate at a maximum temperature of about $200 \mathrm{mK}$ and remain below this limit. To maximize the signal the readout power was operated just a few $\mathrm{dB}$ below bifurcation as discussed above, operating at higher powers has the additional benefit of reducing the TLS noise, $S_{T L S} \propto P_{g}^{-1 / 2}$. Fig. 7(a) shows the measured TLS frequency noise power spectral density for two different temperatures. Two roll-off frequencies are visible. The first appears at $\sim 200 \mathrm{~Hz}$ and is consistent with the resonator bandwidth under no load, where the resonator $Q$ is dominated by the coupling $Q_{c}$. A second roll off appears around $10^{5} \mathrm{~Hz}$ due to anti-alias filtering of the measurement electronics. For frequencies within the resonator bandwidth the noise exhibits a slope above $1 \mathrm{~Hz}, S \propto \nu^{-1 / 2}$, which is characteristic of TLS noise. Increasing the temperature from $60 \mathrm{mK}$ to 
$200 \mathrm{mK}$ decreases the noise approximately as $T^{-2}$. The amplifier noise remains roughly constant in fractional frequency units for these two measurements since $Q_{c}$ dominates the resonator quality factor.
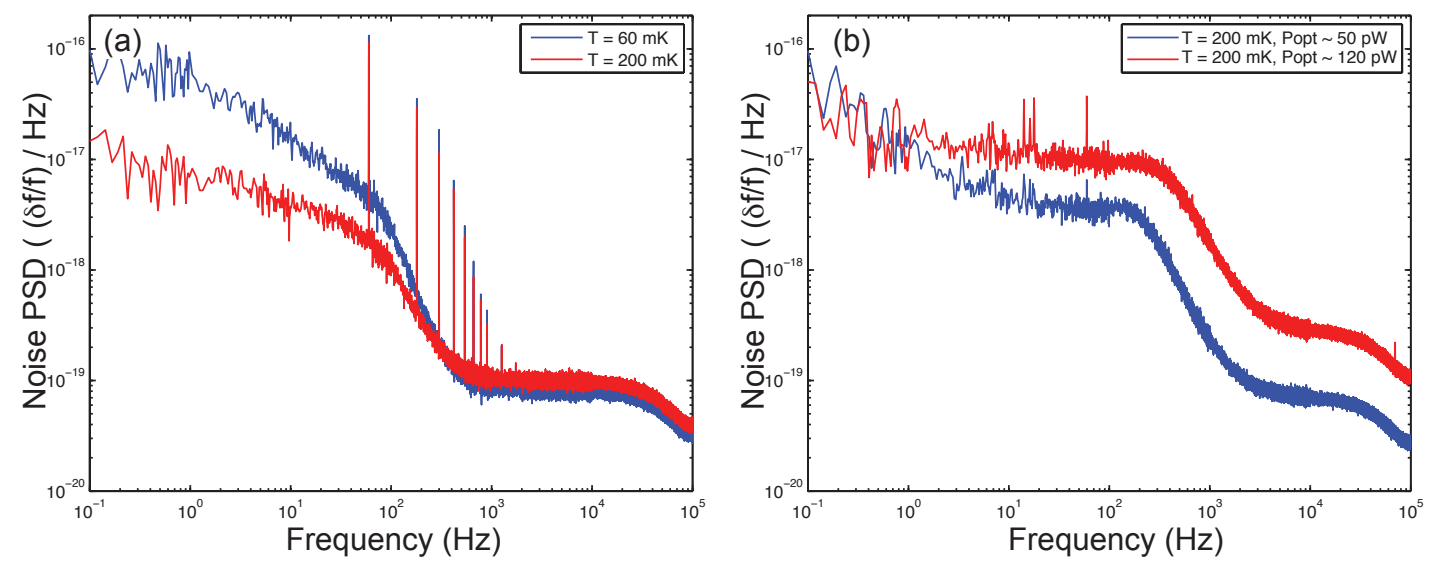

Figure 7. Noise measurements for a $T_{c}=1.2 \mathrm{~K}$ MAKO test pixel in a dilution refrigerator. (a) Measurements done without optical loading at temperatures of $60 \mathrm{mK}$ and $200 \mathrm{mK}$. The spectra show two roll-off frequencies. The first at $\nu \approx 200$ $\mathrm{Hz}$ is due to the resonator bandwidth while the other at $\nu \sim 10^{5} \mathrm{~Hz}$ is due to anti-aliasing filtering in the measurement electronics. Two signatures of TLS noise are present. The first is the slope of the noise, typically TLS noise has slope $\sim \nu^{-0.5}$. The second is that the noise decreases roughly as $T^{-2}$. (b) Measurements done at $200 \mathrm{mK}$ under optical loading. Under the lower power ( $50 \mathrm{pW}$ ) loading the TLS slope is still visible until $\nu \approx 10 \mathrm{~Hz}$, above this the noise is flat until the resonator roll-off. At higher optical power $(120 \mathrm{pW})$ the flat region becomes more dominant at $\nu \approx 1 \mathrm{~Hz}$. The ratio of the values in the flat region of the spectrum within the resonator bandwidth indicate the presence of photon shot noise.

As the blackbody temperature is increased, the shape of the noise spectra changes as shown in Fig. 7(b). The increased optical power reduces the resonator quality factor and increases the resonator bandwidth. The amplifier contribution to the PSD in fractional frequency units begins to increase as $Q_{r}$ decreases, but even at the lowest $Q$ under the highest optical power the noise within the resonator bandwidth is still $\sim 10$ times higher than the measured amplifier noise. Further under blackbody load the the nature of the noise within the resonator bandwidth has changed significantly. At a single-polarization loading of $50 \mathrm{pW}$ the TLS noise appears with its usual decline versus noise frequency until about $10 \mathrm{~Hz}$ where the noise spectra becomes flat and remains so until the resonator roll off. Under a $120 \mathrm{pW}$ loading the flat portion of the spectrum begins near $1 \mathrm{~Hz}$. This flat spectrum is indicative of photon noise. Additionally since photon noise scales as $S_{\delta f / f^{2}} \propto P_{o p t}$ the ratio between these two levels should be $S_{\delta f / f^{2}} \approx 120 / 50=2.4$, almost exactly the ratio seen at $\sim 100 \mathrm{~Hz}$ in Fig. 7 (b). These results indicate that the device is nearly background limited at these optical powers.

\section{CONCLUSIONS}

Based on the measured responsivity and fractional frequency noise, the NEP $(\mathrm{NEP}=\sqrt{S} / R)$ at $1 \mathrm{~Hz}$ under optical load is estimated to be $\sim 3 \times 10^{-16} \mathrm{~W} / \mathrm{Hz}^{-1 / 2}$ which is very nearly the level needed for background limited operation at the CSO. While the measurement of optical responsivity is still subject to a modest uncertainty, the presence of photon noise confirms that we are very nearly background limited in a device without optimized optical efficiency. Simply adding an anti-reflective coating and using a quarter wave backshort will improve the NEP with little effort.

Development of a photon noise limited pixel for operation at $350 \mu \mathrm{m}$ is critical for the future of large focal plane ground based astronomy. We have demonstrated a pixel geometry which is compatible with load readout frequency $\left(f_{\text {res }} \approx 100 \mathrm{MHz}\right)$ and appropriate $Q$ s needed for the MAKO instrument and shown that this pixel can be used in an array format while achieving nearly background limited performance. Additional scaling to larger arrays will also be incorporated in to future designs, as will improvements in the coupling $Q$ and better capacitor design to further decrease the NEP. Looking to the future of sub-millimeter measurements especially 
for CCAT further reductions in the NEP are required. The NEPs of these devices can be easily improved by using lens coupling; the absorber volume could easily be reduced by 1 - 2 orders of magnitude while ensuring that almost all light falling on the focal plane is absorbed. This eliminates the problem of wasted focal plane space in the current design, while also allowing for a much larger capacitor to be used which should decrease the TLS noise further. These improvements coupled with further exploration of the noise properties and physics of TiN should allow substantial reduction in the NEP with very little change to the fundamental design architecture.

\section{ACKNOWLEDGMENTS}

This research was carried out in part at the Jet Propulsion Laboratory (JPL), California Institute of Technology, under a contract with the National Aeronautics and Space Administration. The devices used in this work were fabricated at the JPL Microdevices Laboratory. This work was supported in part by the Keck Institute for Space Science, the Gordon and Betty Moore Foundation, and NASA grant NNX10AC83G. C. McKenney and L. Swenson acknowledge support from the Keck Institute for Space Studies. L. Swenson acknowledges support from the NASA Postdoctoral Program.

\section{REFERENCES}

[1] "Decadal Survey of Astronomy and Astrophysics, Panel Reports - New Worlds, New Horizons in Astronomy and Astrophysics," (2011). http://www.nap.edu/catalog.php?record_id=12982.

[2] Doyle, S., Mauskopf, P., Naylon, J., Porch, A., and Duncombe, C., "Lumped element kinetic inductance detectors," Journal of Low Temperature Physics 151, 530-536 (2008).

[3] Swenson, L. J., Day, P. K., Dowell, C. D., Eom, B. H., Hollister, M. I., Jarnot, R., Kovacs, A., Leduc, H. G., McKenney, C. M., Monroe, R., Morczkowski, A. K., Nguyuen, H. T., and Zmuidzinas, J., "MAKO: A pathfinder instrument for on-sky demonstration of low-cost 350 micron imaging arrays," Ground-based and Airborne Instrumentation for Astronomy III 8452, SPIE (2012).

[4] Gao, J., The Physics of Superconducting Microwave Resonators, PhD thesis, California Institute of Technology (2008).

[5] Leduc, H. G., Bumble, B., Day, P. K., Eom, B. H., Gao, J., Golwala, S., Mazin, B. A., McHugh, S., Merrill, A., Moore, D. C., Noroozian, O., Turner, A. D., and Zmuidzinas, J., "Titanium nitride films for ultrasensitive microresonator detectors," Applied Physics Letters 97, 102509 (2010).

[6] Zmuidzinas, J., "Superconducting microresonators: Physics and applications," Annu. Rev. Cond. Mat. Phys. 3, 15.1-15.46 (2012).

[7] Billot, N., Agnese, P., Augueres, J.-L., Beguin, A., and Bouere, A., "The Herschel/PACS 2560 bolometers imaging camera," Space Telescopes and Instruments I 6265, 626511, SPIE (2006).

[8] Noroozian, O., Day, P. K., Eom, B. H., Leduc, H. G., and Zmuidzinas, J., "Crosstalk reduction for superconducting microwave resonator arrays," IEEE Trans. Micr. Theory Tech. 60, 1235-1243 (2012). 